\title{
Allied health - 3008. Comparison of anti-allergic effect of pneumococcal conjugated vaccine and pneumococcal polysaccharide vaccine in a murine model of house dust mite allergic rhinitis
}

\author{
Soo Whan Kim*, Boo-Young Kim
}

From 2nd WAO International Scientific Conference (WISC 2012)

Hyderabad, India. 6-9 December 2012

\section{Background}

It was discovered that pneumococcal vaccine can reduce the allergic disease. The purpose of the present study is to evaluate the anti-allergic effect of pneumococcal conjugated vaccine and pneumococcal polysaccharide vaccine in a murine model of house dust mite allergic rhinitis. We also evaluated difference between effect of both vaccines.

\section{Methods}

Forty mice were divided into four groups: control, Der f, pneumococcal polysaccharide vaccine (PV), and protein conjugate polysaccharide vaccine (PCV). Allergic rhinitis was induced in BALB/c mice by intraperitoneal sensitization and intranasal challenge with Dermatophagoides farinae (Der $\mathrm{f}$ ). The allergic symptom after the final challenge was recorded. Interferon (IFN)- $\gamma$, Interleukin (IL)-13, and IL-10 levels in nasal lavage fluid (NALF), as well as serum Der f-specific IgE levels were measured. The number of eosinophils in lamina propria was evaluated. The levels of T-bet, GATA-3, and Foxp3 mRNA expression in splenic mononuclear cells were determined by real-time polymerase chain reaction. A comparison of the frequency of $\mathrm{CD} 4^{+} \mathrm{CD} 25^{+}$Foxp $3^{+}$regulatory $\mathrm{T}$ cells in splenic mononuclear cells were made by flow cytometry.

\section{Results}

The T-bet m RNA level was lower in the PV and PCV group than Der f group ( $<<0.05)$. The IL-13, GATA-3 mRNA level and serum Der f-specific IgE and eosinophil were lower in the PV and PCV group than Der f group $(\mathrm{p}<0.05)$. Foxp3 mRNA expression in the PV and PCV group was elevated compared to the Der $\mathrm{f}$ group $(\mathrm{p}<$ $0.05)$. In flow cytometry, the PV group $(\mathrm{p}<0.05)$ and the PCV group $(\mathrm{p}<0.05)$ had higher percentages of CD4+ CD25+Foxp3+ T cells than the Der f group. In the PV group, the percentage of these cells was higher than that in the PCV group. $(\mathrm{p}=0.00)$.

\section{Conclusions}

These results suggest that the pneumococcal polysaccharide vaccines will suppress the allergen-specific Th2 response and enhanced the induction of regulatory $\mathrm{T}$ cells in a model of allergic rhinitis. And the process to work regulatory $\mathrm{T}$ cell can be different between the PV and PCV group.

Published: 23 April 2013

\section{doi:10.1186/1939-4551-6-S1-P184}

Cite this article as: Kim and Kim: Allied health - 3008. Comparison of anti-allergic effect of pneumococcal conjugated vaccine and pneumococcal polysaccharide vaccine in a murine model of house dust mite allergic rhinitis. World Allergy Organization Journal 2013 6(Suppl 1): P184.

The Catholic University of Korea, South Korea 